LETTER TO THE EDITOR

\section{Tracking adverse events in RCTs: lack of agreement among regulatory institutions}

We need to do a better job of keeping track of potential side effects when designing rand omized clinical trials (RCTs). Consider an RCT for a new drug tested for the main or first order effect-the reduction of hypertension. Power calculations are carried out so that meaningful differences between the drug users and the controls can be detected on this effect. Second order effects-in this case, mortality-are those adverse events that are prospectively tracked, recorded, planned for in advance and, if sufficiently serious, may bring the trial to a halt through review conducted by a Data Safety Monitoring Board. Too rarely are there systematic prospective efforts to collect information on other unpredicted effects of the drug being tested. These third order effects may be important but are rare and unexpected and are not therefore necessarily sought out, so systematic data on them are not prospectively collected. This problem of failing to track such third order events is vividly shown in the recent article about Bruce Psaty and his recognition of the third order side effects related to the use of calcium channel blockers.

Although the US Food and Drug Administration (FDA) and other Government agencies have systems in place for the post-marketing reporting of possible side effects, this surveillance is passive in nature and relies mainly on the judgment of practitioners to suggest whether or not a patient's complaint is related to the drug in question. This creates an inherent underreporting of side effects. It also assumes that everyone has the same access to health care or that, by the time the complaint is reported, there is enough time to avoid serious complications in other patients.

The US National Institutes of Health (NIH) and the World Health Organization (WHO) are aware of these issues. NIH guidelines appear to be focused on multicenter clinical trials; the definition of adverse events is less well characterized for single center trials. Furthermore, the definition of an adverse event, while in compliance with the FDA regulations, is somewhat broad or vague. For example, the federal regulation requires the reporting of adverse events that are "serious and unexpected". This term could be interpreted differently. Does the event in question need to be both serious and unanticipated by the investigator? What about unanticipated effects regardless of the seriousness? How serious? Are events that are costly to a patient in terms of productive time, money, or psychological damage considered serious? A summary of the NIH Institutes and their guidelines and websites is shown in table 1 .

This lack of uniformity and clarity is not peculiar to the USA. Some international institutions rely on the WHO to establish their adverse reporting mechanism standards. In their guidelines for good clinical practice GCP) for trials on pharmaceutical products the WHO states that the investigator is responsible for notifying (with documentation) the relevant health authorities, the sponsor and, when applicable, the ethics committee immediately in the case of serious adverse events or reactions, as governed by national regulations . .". This statement contains little guidance as to who is the relevant authority, what documentation to use, and under what circumstances the established ethical committee should be involved. Furthermore, the statement "as governed by national regulations" does not clearly assign responsibility for the reporting of adverse events, especially in situations when one nation is conducting medical trials in another country. Moreover, the WHO falls short in suggesting any type of analyses for the examination of possible population wide repercussions.

Countries in Central and South America basically follow the guidance of the WHO through the Pan-American Health Organization (PAHO). In England and Australia, information about adverse events, especially if observed after the trials and/or the medication is on the open market, is not easily accessible.

We think there should be more clarity and uniformity in these guidelines and that all guidelines should be accessible through the internet or some other form of common global communication to the scientific community. Furthermore, investigators and agencies alike should be aware of studies reporting

Table 1 Procedures for monitoring adverse events for USA NIH Institutes

\begin{tabular}{|c|c|c|}
\hline Agency & Procedure & Web site \\
\hline $\mathrm{NIH}$ & $\begin{array}{l}\text { General guidelines for all institutes and centers (IC). Requires } \\
\text { establishment of Data Safety Monitoring Boards (DSMB) for multisite } \\
\text { clinical trials. Gives each IC the ability to establish its own procedures. }\end{array}$ & http://grants 1.nih.gov/grants/guide/index.hml/ \\
\hline $\mathrm{NCl}$ & $\begin{array}{l}\text { Follows general NIH guidelines. Additionally, defines "adverse events" } \\
\text { and gives categories for severity and attribution of such events }\end{array}$ & http://ctep.info.nih.gov. \\
\hline NEI & Follows general NIH guidelines. & http://wwwt.nei.nih.gov/funding/policy6.htm. \\
\hline $\mathrm{NHLBI}$ & $\begin{array}{l}\text { Follows general NIH guidelines. Specifies situations where the primary } \\
\text { investigator submits safety monitoring plans. }\end{array}$ & http://rover2.nhlbi.nih.gov/policies \\
\hline NHGRI & Issue does not appear to be specifically addressed & http://www.nhgri.nih.gov/ \\
\hline NIA & Follows general NIH guidelines. & http://www.nia.nih.gov/funding/policies/gfunding.html\#data \\
\hline NIAAA & Issue does not appear to be specifically addressed & http://www.niaaa.nih.gov/ \\
\hline NIAID & Gives guidelines for DSMBs & http://www.niaid.nih.gov/dmid/clinresearch/dsm.htm \\
\hline NIAMS & Follows general NIH guidelines. & http://www.niams.nih.gov/rtac/clinical/safe_monitoring_plan.htm \\
\hline NIBIB & Issue does not appear to be specifically addressed & http://www.nibibl .nih.gov/ \\
\hline $\mathrm{NICHD}$ & Follows general NIH guidelines & http://www.nichd.nih.gov/funding/datasafety.htm \\
\hline NIDCD & Follows general NIH guidelines. & http://www.nided.nih.gov/funding/clinical/clin_policy.htm \\
\hline NIDCR & Follows general NIH guidelines. & http://www.nidr.nih.gov/research/ \\
\hline NIDDK & $\begin{array}{l}\text { Follows general NIH guidelines. Additionally, defines "adverse events" } \\
\text { and gives categories for severity, attribution, action taken and } \\
\text { outcome of such events. }\end{array}$ & http://www.niddk.nih.gov/patient/patient.htm\#policy \\
\hline NIDA & Gives detailed guidelines for DSMBs & http://www.nida.nih.gov/Funding/DSMBSOP.html \\
\hline NIEHS & Follows general NIH guidelines. & http://www.niehs.nih.gov/dert/clinsafe.htm \\
\hline NIGMS & Follows general NIH guidelines & http://www.nigms.nih.gov/ \\
\hline $\mathrm{NIMH}$ & Gives detailed guidelines for DSMBs & http://www.nimh.nih.gov/research/safetymonitoring.cfm \\
\hline NINDS & Institute appoints Performance and Safety Monitoring Boards & http://www.ninds.nih.gov/funding/ninds_patient_safety_guidelines.htm \\
\hline NINR & Issue does not appear to be specifically addressed & http://www.nih.gov/ninr/ \\
\hline
\end{tabular}

$\mathrm{NIH}=$ National Institutes of Health; $\mathrm{NCl}=$ National Cancer Institute; $\mathrm{NEI}=$ National Eye Institute; $\mathrm{NHLBI}=\mathrm{National} \mathrm{Heart,} \mathrm{Lung} \mathrm{and} \mathrm{Blood} \mathrm{Institute}$ NHGRI=National Human Genome Research Institute; NIA=National Institute on Aging; NIAAA=National Institute on Alcohol Abuse and Alcoholism; NIAID=National Institute of Allergy and Infectious Diseases; NIAMS=National Institute of Arthritis and Musculoskeletal Diseases; NIBIB=National Institute of Biomedical Imaging and Bioengineering; NICHD=National Institute of Child Health and Human Development; NIDCD=National Institute on Deafness and Other Communication Disorders; NIDCR=National Institute of Dental and Craniofacial Research; NIDDK=National Institute of Diabetes and Digestive and Kidney Diseases; NIDA=National Institute on Drug Abuse; NIEHS=National Institute of Environmental Health Sciences; NIGMS=National Institute of General Medical Sciences; NIMH=National Institute of Mental Health; NINDS=National Institute of Neurological Diseases and Stroke; NINR=National Institute of Nursing Research. 
adverse events. These reporting mechanisms should have direct relationship with post marketing surveillance efforts. ${ }^{3}{ }^{4}$

L M Santiago, S M Debanne, D Neuhauser Department of Epidemiology and Biostatistics, Medical School, Case Western Reserve University, Cleveland, Ohio 44106-4945, USA

Correspondence to: Dr L M Santiago, Epidemiology and Biostatistics, Medical School, Case Western Reserve University, Cleveland, Ohio 44106-4945, USA; santiago@neo.cwru.edu.

\section{References}

1 Deyo RA. Bruce Psaty and the risks of calcium channel blockers. Qual Saf Health Care 2002;1 1:294-6.

2 World Health Organisation. Guidelines for good clinical practice (GCP) for trials on pharmaceutical products. Geneva: World Health Organisation, 1995: Annex 3.

3 Begg C, Cho M, Eastwood S, et al. Improving the quality of reporting of randomized controlled trials. The CONSORT statement JAMA 1996;276:637-9.

4 Tramer MR, et al. Quantitative estimation of rare adverse events which follow a biological progression: a new model applied to chronic NSAID use. Pain 2000;85:169-82.

\section{BOOK REVIEWS}

\section{Improving Patient Safety:} Insights from American, Australian and British healthcare

Emslie S, Knox K, Pickstone M, eds. London: ECRI and Department of Health, 2002 £35.00. 104 pp. ISBN 0941417751

What happened when a medical devices officer decided to explore the cupboards at his hospital is one of the most attention grabbing parts of this book. Chris Quinn, from Newcastle upon Tyne Hospitals NHS Trust in the UK, found more than 40 kinds of infusion pumps, 25 of which were obsolete, and discovered that no nurse had received competency based training in their use. An increase in infusion incidents between 1993 and 1996 has now been reversed at the Trust thanks to a training programme and the growth of what Mr Quinn describes as "a culture of safety".

The theme of a safety culture runs through the book, which takes the form of abridged transcripts of speakers' presentations at a conference to introduce the UK's National Patient Safety Agency (NPSA) in 2001. It is edited by the speakers and is therefore an accurate account of the day's events. It contains some good material.

Since the publication of An Organisation with a Memory in 2000 there has been an increasing emphasis on patient safety in the UK's NHS, and the first few presentations in the book are therefore concerned with initial steps in this direction and with the launch of the NPSA. For example, the then head of controls assurance at the Department of Health, Stuart Emslie, outlines the new national system for learning from adverse incidents in the context of the governance and controls assurance agenda. He adds that one could argue that underinvestment in health service management is a significant factor in the high incidence of preventable harm to patients. As the previous speaker, health minister Lord Hunt, points out, this costs the taxpayer money: prolonged hospital stays due to adverse events cost the NHS at least £2bn a year.

Presentations from America, Australia and New Zealand add an international dimension to the book. Dr Paul Barach from the University of Chicago's Center for Patient Safety talks of improving patient safety by "replacing the fragmented approach by teamwork, and inviting the patient into the system", while director of the Veterans' Health Administration's National Center for Patient Safety, Dr James Bagian, warns of the potentially dire consequences of failing to protect the confidentiality of those making reports. He says that the civil aviation authorities in New Zealand once revealed the name of a captain who made a report: as a result their system lost the trust of the aviation community and was disbanded.

The book carries case studies and examples from a variety of health systems, and these will perhaps be of most interest to readers trying to improve patient safety day-to-day. Professor Bill Runciman, President of the Australian Patient Safety Foundation, discusses improvements in prescribing of nonsteroidal anti-inflammatory drugs (NSAIDs) and the use of pulse oximeters, while exam ples from the UK such as Professor Nick Barber's work on medication errors and the work of Chris Quinn are particularly likely to resonate with the home audience.

All in all, the book adds to the background knowledge about patient safety and is particularly strong when it talks about solutions to patient safety problems. Some parts are starting to look dated-for example, where the work of the NPSA is concerned-as there have been many developments since October 2001 , but overall it is a valuable read for those thinking about and implementing patient safety measures.

Pat Anderson

Editor, Health Care Risk Report, Butterworths Tolley pat@leonardal.freeserve.co.uk

\section{Take Heart! A Guide to Coping with Cardiac Surgery for Patients} and their Families

Maclaughlan $M$, ed. Librario. (Pp 122; £7.99). ISBN 0954296087

A small book, this, but packed as full of meat as an egg. There is not a line wasted in MacLachlan's account of how patients face up to, go through, and recover from the experience of cardiac surgery.

What patients? A statistician might complain that there is no evidence in these pages of studies made by experts of carefully balanced statistically valid groups (including control groups, of course), no elegantly designed charts or tables-nothing, in fact, to add to any academic's collection of important texts. Just a lot of chat, really. Which is what makes this book so important. It is chat. It is an account of patients talking to each other, either directly or in quotes from letters, about their experiences of cardiac operations.

As I read it, it suddenly became intensely familiar to me. It transported me back to my years nursing on acute surgical wards, hearing my patients gossip to each other about their feelings and their past experiences and future plans, at the same time as taking care of their nervous bed mates by reassuring the anxious, helping the most ill by being suitably in awe of one who had had an unusual experience, and generally creating a sort of brother/sisterhood of patients.

This book manages to provide the same comfortable easy attitude even though it threw its net wide; patients from the USA and Canada as well as the UK provided information about their experiences and, above all, about how they felt about what happened to them.

Thus, there is discussion of painbackache, pain in the legs from the site of removal of the veins used in the cardiac surgery (both apparently much worse than the pain from the chest operation site)-but with it clear appreciation of the fact that medical and nursing staff are not nowadays stingy with analgesics, and that some pain is not susceptible to them and must simply be lived with until it decides to fade away in its own time.

The simplicity of this extremely successful little book with its direct cheerful tone, together with all the useful information it provides about sources of further information, made me wonder why it is that the Government appears at present to be so anxious about ensuring patient involvement with all aspects of NHS practice, leading to the setting up of Patients' Forums and the like. What is needed is not formal bodies like Patients' Forums with their minutes and subcommittees and all the other paraphernalia so popular in today's highly structured Harvard Business School influenced NHS, but access to clear unvarnished patient opinion at source.

There is no suggestion anywhere in this book that Ms MacLachlan is an experienced writer or communicator or anything other than a warm observant human being with a lively interest in what happens to her and to people like her who share her experiences. Surely there are Ms MacLachlans all over the country in the NHS (or private wards; the author was not shy about including such patients in her review) who need simply to be identified (most experienced senior ward sisters would be able to do that) and given the opportunity to produce just such a text as this, but dealing with orthopaedic surgery, gynaecological surgery, or whatever.

It would certainly cost far less than the current efforts to "empower patients" — and I strongly suspect it would be a great deal more effective.

Claire Rayner clairerayner@harrowhill.demon.co.uk 